\title{
Spin vector orientations of galaxies in seven Abell clusters of BM type III
}

\author{
B. Aryal and W. Saurer
}

\author{
Institut für Astrophysik, Universität Innsbruck, Technikerstraße 25, 6020 Innsbruck, Austria \\ e-mail: [Binil.Aryal; Walter.Saurer]@uibk.ac.at
}

Received 8 September 2004 / Accepted 19 November 2004

\begin{abstract}
We present an analysis of the spatial orientations of 851 galaxies in 7 Abell clusters of BM type III (type III in the Bautz-Morgan system). Anisotropy is found in all 7 clusters. It is found that the spin vector orientations of the galaxies in the clusters of BM type III tend to be oriented both parallel (Abell 1412, Abell 2048 and Abell 4038) and perpendicular (Abell 2061, Abell 2065, Abell 2151 and Abell 2197) to the local supercluster plane. The spin vector projections of galaxies in 5 clusters (Abell 2061, Abell 2065, Abell 2151, Abell 2197 and Abell 4038) is found to be oriented perpendicular to the Virgo cluster center.
\end{abstract}

Key words. galaxies: evolution - galaxies: formation - galaxies: statistics - galaxies: clusters: general

\section{Introduction}

In a previous paper (Aryal \& Saurer 2004, hereafter Paper I) we presented the spatial orientations of 1231 galaxies in 8 Abell clusters of BM type I (type I in Bautz-Morgan system, Bautz \& Morgan 1970). The investigated clusters were Abell 42, Abell 401, Abell 1775, Abell 2199, Abell 3556, Abell 3558, Abell 3560 and Abell 3562. No preferred alignment is found in Abell 401, Abell 2199 and Abell 3556. In other clusters (Abell 42, Abell 1775, Abell 3558, Abell 3560 and Abell 3562) we noticed different alignments.

In this paper we analyse the spatial orientations of 851 galaxies in 7 Abell clusters of BM type III. The BM type III designates open, late-type, irregular, asymmetrical and spiralrich clusters. Since spiral galaxies are supported by rotation, a study of galaxy alignment in these spiral-rich clusters is important (Kashikawa \& Okamura 1992). These clusters are also important in galaxy orientation studies because of their rather low X-ray luminosity and radio emission (Andernach \& Andreazza 1990; van den Bergh 1978). The SV orientations of galaxies in these clusters can be an indicator of initial conditions when galaxies and clusters formed provided the angular momenta of galaxies have not been altered too much since their formation. A useful property of galaxies in clusters for which theories make different predictions is the angular momentum distribution. The "Pancake model" by Doroshkevich (1973), the "Hierarchy model" by Peebles (1969) and, the "Primordial vorticity model" by Ozernoy (1978) predict different scenarios concerning the formation of large-scale structure. Thus, the study of galaxy orientation has the potential to yield important information regarding the formation and evolution of cosmic structures.

We use similar methods, statistics and data reduction procedure as in Paper I. The investigated clusters are Abell A1412, Abell 2048, Abell 2061, Abell 2065, Abell 2151, Abell 2197, and Abell 4038. The radial velocities of these clusters are very large (radial distances $\sim 300 \mathrm{Mpc}$ ). Therefore a relation of the orientation of these galaxies with respect to the Local Supercluster or the Virgo cluster is unexpected.

In the past only a few investigations have been carried out to study the orientation of galaxies in the clusters of BM type III. Baier et al. (2003) published their study of galaxy orientations in Abell 14. They found strong evidence for the alignment of galaxy position angles (PAs) in the clusters in the sense that the major planes of galaxies tend to be parallel to the direction of the PA of the major axis of the cluster. Hu et al. (1995) studied the orientation of 310 disk galaxies in the Virgo cluster and found anisotropy. Adams et al. (1980) included two BM type III clusters (Abell 179 and Abell 2197) in their dataset out of seven Rood-Sastry L-type Abell clusters in a study of galaxy alignment. Anisotropy is found in the PA distribution of galaxies in these two clusters. The alignment of galaxies was found to very prominent in Abell 2197. Thompson (1976) studied the majoraxis PAs of galaxies in four BM type III clusters (Virgo cluster, Abell 2147, Abell 2151 and Abell 2197) and found anisotropy in Virgo and Abell 2197. Abell 2147 and Abell 2151 showed isotropy.

After describing the sample used and the method of data reduction in Sect. 2, we discuss the methods, statistical tools and 
Table 1. Data of our sample. The first three columns list the Abell number and the position of the cluster center $(\alpha(2000), \delta(2000))$. The next columns give radial velocity $(R V)$, distance class $(D)$, richness class $(R)$ and the red magnitude of the tenth brightest cluster member $\left(m_{10}\right)$ as listed in the ACO catalogue. The last two columns give the total number of galaxies investigated (by the authors) in the cluster region and the galaxies with known position angle, respectively.

\begin{tabular}{lcccccccc}
\hline \hline Abell & $\alpha(2000)$ & $\delta(2000)$ & $\begin{array}{c}R V \\
\left(\mathrm{~km} \mathrm{~s}^{-1}\right)\end{array}$ & $D$ & $R$ & $m_{10}$ & $N_{T}$ & $N$ \\
\hline 1412 & $11^{\mathrm{h}} 55^{\mathrm{m}} 45^{\mathrm{s}} .2$ & $+73^{\circ} 28^{\prime} 18^{\prime \prime}$ & 25003 & 4 & 2 & 15.9 & 156 & 130 \\
2048 & $15^{\mathrm{h}} 15^{\mathrm{m}} 17.8$ & $+04^{\circ} 22^{\prime} 56^{\prime \prime}$ & 28330 & 4 & 1 & 16.0 & 121 & 104 \\
2061 & $15^{\mathrm{h}} 02^{\mathrm{m}} 15^{\mathrm{s}} 3$ & $+30^{\circ} 39^{\prime} 17^{\prime \prime}$ & 23024 & 4 & 1 & 15.7 & 127 & 97 \\
2065 & $15^{\mathrm{h}} 22^{\mathrm{m}} 42^{\mathrm{s}} .6$ & $+27^{\circ} 43^{\prime} 21^{\prime \prime}$ & 21765 & 3 & 2 & 15.6 & 188 & 144 \\
2151 & $16^{\mathrm{h}} 05^{\mathrm{m}} 15^{\mathrm{s}} .0$ & $+17^{\circ} 44^{\prime} 55^{\prime \prime}$ & 10943 & 1 & 2 & 13.8 & 190 & 172 \\
2197 & $16^{\mathrm{h}} 28^{\mathrm{m}} 10^{\varsigma} .4$ & $+40^{\circ} 54^{\prime} 26^{\prime \prime}$ & 9223 & 1 & 1 & 13.9 & 117 & 102 \\
4038 & $23^{\mathrm{h}} 47^{\mathrm{m}} 31^{\mathrm{s}} .1$ & $-28^{\circ} 12^{\prime} 10^{\prime \prime}$ & 8994 & 2 & 2 & 13.4 & 126 & 102 \\
\hline
\end{tabular}

the selection effects in Sect. 3. A discussion of the statistical results and the conclusions are presented in Sects. 4 and 5.

\section{The sample}

The selection criteria for the clusters were identical to those used in Paper I: (1) the morphology of the cluster should be "type III" in the BM system; (2) the clusters need to have an Abell richness $\geq 1$ (Abell et al. 1989, hereafter ACO); (3) the clusters need to fall outside the galactic plane $\left(b^{\mathrm{II}}>30^{\circ}\right)$; (4) the radial velocities (RVs) of the clusters should be known; and (5) the number of galaxies in the cluster should be $>100$. We inspected 29 clusters fulfilling the selection criteria on the film copies (red sensitive ESO/POSS II) with the aid of a binocular microscope (25-fold magnification) and selected 7 of them: the galaxies in these clusters were not too faint and it was possible to determine diameters and PAs of many galaxies with high accuracy.

The method of investigation and the measurements of positions, PAs and diameters $(a, b)$ were the same as in Paper I.

A list of the investigated Abell clusters is given in Table 1. The positions of galaxies in these clusters are shown in Fig. 1. The galaxies with known PAs are represented by a plus (+) and those with unknown PAs by a hollow triangle $(\triangle)$. Solid lines in Fig. 1 represent the cluster PA as defined by Rhee \& Ross (1989). The cluster centers are located at the centers of the circles shown in Fig. 1.

It is estimated that the background contamination of galaxies in the investigated cluster region is 9-20\%. This estimation was based on the area distribution of background galaxies around the cluster region.

The foreground field galaxies in the investigated cluster region have been removed with the help of the Uppsala General Catalogue of Galaxies (Nilson 1973), the Third Reference Catalogue of Bright Galaxies (de Vaucouleurs et al. 1991), the ESO/Uppsala Survey of the ESO (B) Atlas (Lauberts 1982), and the Morphological Galaxy Catalogue (Vorontsov-Vel'Yaminov et al. 1962-74).

\section{Method of analysis}

We follow the "position angle - inclination" method (Flin \& Godlowski 1986) to derive spatial orientations of spin vectors
(SVs) of galaxies. The three-dimensional orientation of the SV of a galaxy is characterized by two angles: the polar angle $(\theta)$ between the galactic SV and a reference plane, and the azimuthal angle $(\phi)$ between the projection of a galactic SV on to the reference plane and the $X$-axis within this plane. The reference plane is not easy to define in these clusters. We use the Local Supercluster (LSC) plane as defined by Tammann \& Sandage (1976) as a reference for the analysis. We assume a spatial isotropic distribution of SVs of galaxies as a theoretical reference. This spatial reference distribution will give further reference distributions for the angles $\theta$ and $\phi$.

Our observations are compared with expected isotropic distribution curves in both $\theta$ and $\phi$. For this comparison we use three different statistical tests: chi-square, Fourier and autocorrelation. For a detailed description of these tests see Godlowski (1993, 1994). The conditions for anisotropy are the following: the chi-square probability $P\left(>\chi^{2}\right)<0.050$, correlation coefficient $C / \sigma(C)>1$, first order Fourier coefficient $\Delta_{11} / \sigma\left(\Delta_{11}\right)>1$ and the first order Fourier probability $P\left(>\Delta_{1}\right)>0.150$ as used by Godlowski (1993).

Any selection criteria imposed on the data may cause severe changes in the shapes of the expected isotropic distribution curves (Aryal \& Saurer 2000, 2001). The lack of knowledge of PAs of nearly face-on galaxies and an inhomogenous distribution of positions of galaxies are the main selection effects (Baier et al. 2003; Aryal \& Saurer 2005). It is essential to remove both the positional and inclination effect from the database. To do this we use the method described by Aryal \& Saurer (2000).

To remove the Holmberg effect we follow the method of Fouque \& Paturel (1985) to convert the measured diameters to standard photometric diameters. It is found that our data needs an additive constant 0.18 to the visual diameters to bring them on to the photometric system. The errors in $i, \theta$, and $\phi$ are estimated to be $7^{\circ}, 13^{\circ}$ and $18^{\circ}$, respectively. The method for these estimations is described in Paper I.

\section{Results}

We first give the results of the two-dimensional analysis (equatorial PA-distribution) and then discuss its significance for a three-dimentional analysis ( $\theta$ and $\phi$-distribution). 

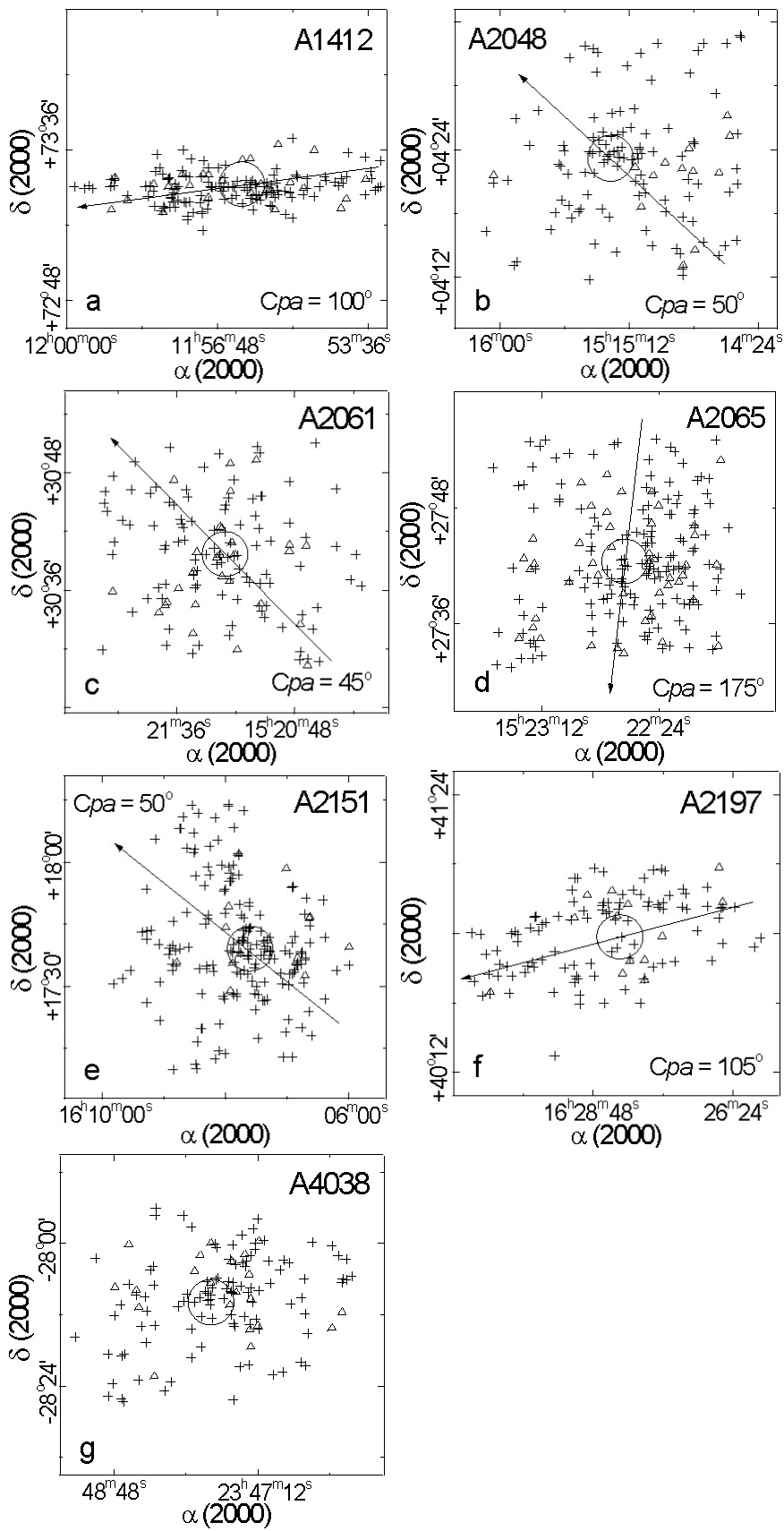

Fig. 1. Distribution of galaxies in the clusters A1412 a); A2048 b); A2061 c); A2065 d); A2151 e); A2197 f); and A4038 g). A plus (+) sign represents a galaxy with known PA. The PA is not known for the galaxies represented by a hollow triangle $(\triangle)$. The cluster center as given in ACO catalogue is at the center of the circle in each figure. The solid line with an arrow represents the position angle of the cluster (Cpa) as defined by Rhee et al. (1991).

\subsection{Anisotropy in the position angle distribution}

We first analyse the distribution of the PAs of the major diameters of cluster galaxies using chi-square and autocorrelation tests. Figure 2 shows the PA-distribution of galaxies in the investigated cluster. The humps and the dips in the histograms of the PA-distribution are listed in Table 2.
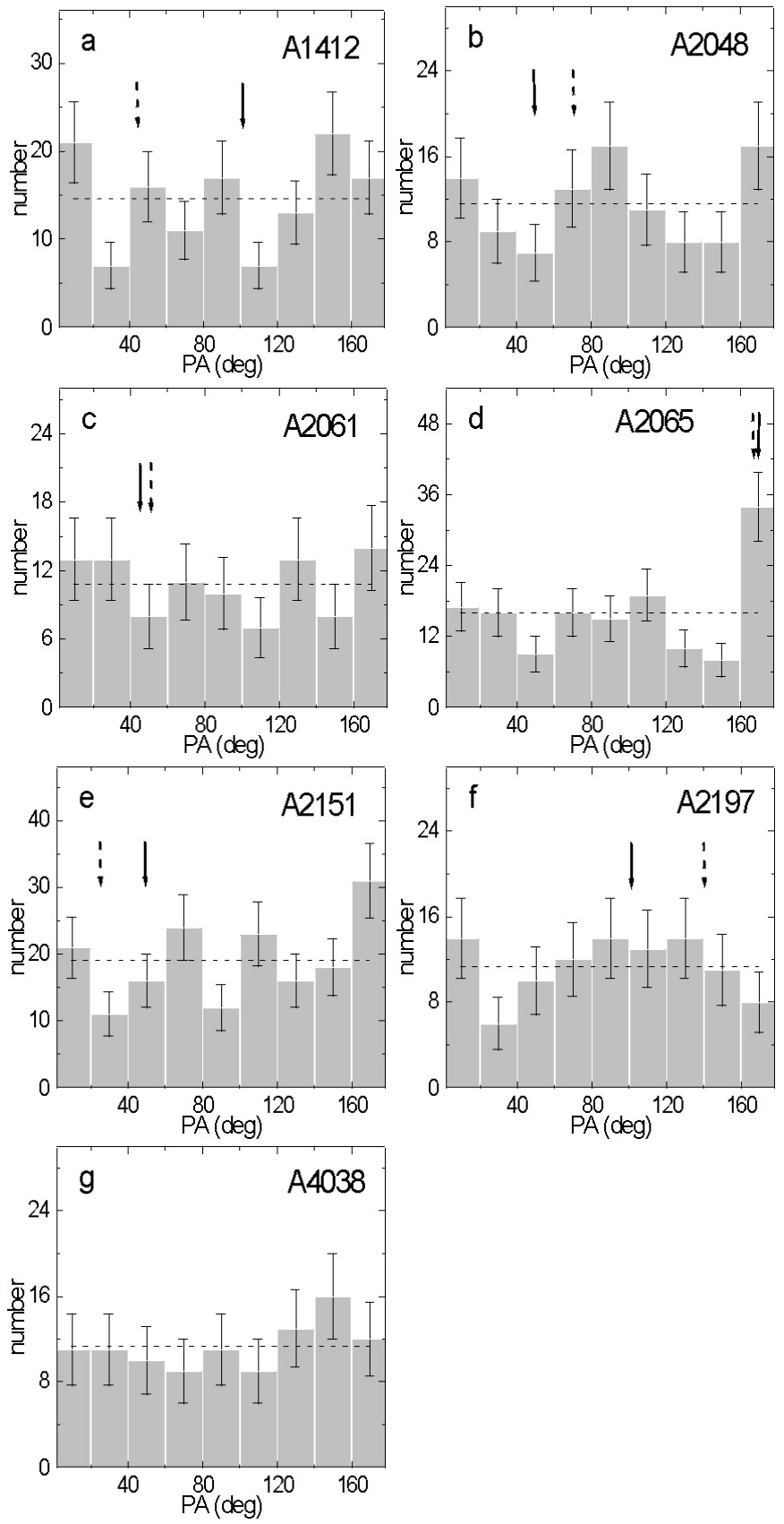

Fig. 2. The equatorial PA distributions of galaxies in the clusters A1412 a); A2048 b); A2061 c); A2065 d); A2151 e); A2197 f); and A4038 g). The dashed line represents the expected distributions. The observed counts with statistical $\pm \sigma$ error bars are shown. $\mathrm{PA}=0^{\circ}$ or $180^{\circ}$ corresponds to the galactic rotation axes lying in the equatorial plane. The solid and dashed arrow represent the cluster PA and the PA of the first-ranked galaxy as listed in Rhee \& Ross (1989) and Kampen \& Rhee (1990), respectively.

The clusters Abell 2048, Abell 2061, Abell 2197 and Abell 4038 show isotropy in both statistical tests. No deviation from an isotropic PA-distribution curve is observed in these clusters.

Three clusters (Abell 1412, Abell 2065 and Abell 2151) show anisotropy in the statistical tests. In the PA-distribution of Abell 1412, humps at $10^{\circ}$ and $150^{\circ}$ and dips at $30^{\circ}$ and $110^{\circ}$ can be seen (Fig. 2a, Table 2). The humps at $10^{\circ}$ and $150^{\circ}$ 
Table 2. Statistics of the PA-distribution of galaxies in 7 Abell clusters of BM type III. The first four columns list the Abell number, cluster PA as listed in Rhee \& Ross (1989), PA of the first-ranked galaxy as listed in van Kampen \& Rhee (1990), elongation strength (high for highly elongated clusters) as listed in Rhee et al. (1991). The next two columns give the dips and humps in PA-distribution (Fig. 2). The next columns give the chi-square probablity and the correlation coefficient.

\begin{tabular}{lcclllll}
\hline \hline Abell & Clu. & f.r.g. & Elo. Dips & Humps & $P\left(>\chi^{2}\right)$ & $C / C(\sigma)$ \\
& PA & PA & str. & $\left(^{\circ}\right)$ & $\left(^{\circ}\right)$ & & \\
\hline 1412 & 100 & 45 & 2.5 & 30,110 & 10,150 & 0.036 & -1.7 \\
2048 & 50 & 73 & 0.8 & 50 & 150 & 0.238 & +0.1 \\
2061 & 45 & 55 & 1.5 & - & - & 0.741 & -0.7 \\
2065 & 175 & 174 & 2.3 & $50,130,150$ & 170 & 0.000 & -2.4 \\
2151 & 50 & 25 & 1.5 & 30,90 & 170 & 0.023 & -1.6 \\
2197 & 105 & 141 & 2.0 & 30 & - & 0.667 & +0.2 \\
4038 & - & - & - & - & - & 0.910 & +0.4 \\
\hline
\end{tabular}

suggest that the PAs of galaxies in this cluster tend to lie in the equatorial plane. Abell 2065 and Abell 2151 both show a significant hump at $170^{\circ}$ in the PA-distribution (Figs. 2d, e) suggesting also that the PAs of galaxies in these clusters tend to be oriented parallel to the equatorial plane.

To understand the evolutionary scenario of a cluster, a good knowledge of its reference system is needed. Van Kampen \& Rhee (1990) studied the nature of the distribution of PAs for the 10-20 brightest galaxies in a sample of rich Abell clusters to estimate the cluster PA. They studied the clusters Abell 1412, Abell 2048 Abell 2061, Abell 2065, Abell 2151, and Abell 2197 and estimated their PAs (second column in Table 2). A strong alignment is noticed in Abell 2065 (see Fig. 2d). It is found that the PAs of galaxies in Abell 2065 tend to lie along the cluster PA. The cluster PA, the PA of the firstranked galaxy and the preferred alignment of galaxies are approximately along the same direction (i.e., 170 ${ }^{\circ}$ ) in Abell 2065. No such alignment is noticed in other clusters.

A strong alignment is noticed in the clusters having elongation strength $>2$ (Abell 1412 and Abell 2065, see Table 2). Thus, anisotropy is found for the highly elongated clusters.

We compare our results of the two-dimensional analysis (PA distribution) and three-dimensional analysis $(\theta$ and $\phi$ distribution) in Sects. 4.2 and 4.3.

\subsection{Anisotropy in the polar angle distribution}

Table 3 lists the statistical parameters for the polar angle $(\theta)$ distribution of the cluster galaxies. Figure 3 shows the polar angle distribution of galaxies in the 7 Abell clusters with respect to the LSC plane.

All three statistical results show anisotropy in the 7 Abell clusters (Table 3). It should be noted that humps (dips) at smaller angles $\left(<45^{\circ}\right)$ and dips (humps) at larger angles $\left(>45^{\circ}\right)$ in the $\theta$-distribution lead to a negative (positive) $\Delta_{11}$ value, suggesting that the SVs of galaxies tend to be oriented parallel (perpendicular) to the LSC plane. In the Fourier test the $\Delta_{11}$ value is found to be negative for Abell 1412, Abell 2048
Table 3. Statistics of the polar angle distribution of galaxies in 7 Abell clusters. The first column lists the Abell number. The next columns give the chi-square probablity, correlation coefficient, first order Fourier coefficient and first order Fourier probablity.

\begin{tabular}{ccccc}
\hline \hline Abell & $P\left(>\chi^{2}\right)$ & $C / C(\sigma)$ & $\Delta_{11} / \sigma\left(\Delta_{11}\right)$ & $P\left(>\Delta_{1}\right)$ \\
\hline 1412 & 0.020 & +1.9 & -1.9 & 0.039 \\
2048 & 0.013 & +2.3 & -1.3 & 0.091 \\
2061 & 0.001 & +3.7 & +2.7 & 0.007 \\
2065 & 0.000 & +3.5 & +1.8 & 0.025 \\
2151 & 0.011 & +2.4 & +1.3 & 0.117 \\
2197 & 0.017 & +3.1 & +1.2 & 0.124 \\
4038 & 0.000 & +3.4 & -2.4 & 0.016 \\
\hline
\end{tabular}

and Abell 4038 and positive for the Abell 2061, Abell 2065, Abell 2151 and Abell 2197.

In Abell 1412 a hump at $25^{\circ}(2 \sigma)$ and a dip at $65^{\circ}(\sim 3 \sigma)$ and $75^{\circ}(\sim 2 \sigma)$ can be seen (Fig. 3a). These humps and dips make the $\Delta_{11}$ value negative at the $1.9 \sigma$ level suggesting that the SVs of galaxies in Abell 1412 tend to lie parallel to the LSC plane.

Similar to Abell 1412, Abell 2048 gives a negative $\Delta_{11}$ value at $1.3 \sigma$ level (Table 3 ). The negative $\Delta_{11}$ value is due to the hump at $15^{\circ}(1.5 \sigma)$ and dip at $45^{\circ}(1.5 \sigma)$ and $55^{\circ}(>3 \sigma)$ (Fig. 3b). We have not taken into account the bin $\theta=85^{\circ}$ because of poor statistics. Thus, the SVs of the galaxies in Abell 2048 tend to be oriented parallel to the LSC plane.

The third cluster which gives a negative $\Delta_{11}$ value in the Fourier statistics is Abell 4038. The negative $\Delta_{11}$ value is due to the hump at $25^{\circ}(2.5 \sigma)$ and $35^{\circ}(1.5 \sigma)$, and the dip at $55^{\circ}(2 \sigma)$ and $65^{\circ}(2 \sigma)$ (Fig. $3 \mathrm{~g}$ ). This suggests that the SVs of galaxies in Abell 4038 tend to lie parallel to the LSC plane.

The four clusters Abell 2061, Abell 2065, Abell 2151 and Abell 2197 show a very similar orientation in the $\theta$-distribution. In all these clusters a hump at $75^{\circ}(>1.5 \sigma)$ and $85^{\circ}(>1.5 \sigma)$ can be seen (Figs. 3c-f). In addition, a dip at $35^{\circ}$ in Abell 2061 and a dip at $45^{\circ}$ and $55^{\circ}$ in Abell 2065 can also be seen (Figs. 3c, d). These humps and dips cause the $\Delta_{11}$ value to be positive which suggests that the SVs of galaxies in these clusters tend to be oriented perpendicular to the LSC plane.

Figures $4 \mathrm{a}-\mathrm{g}$ shows the plot of the SVs of galaxies in the investigated clusters. These figures are plotted in the supergalactic coordinate system: $L$ and $B$ represent supergalactic longitude and latitude, respectively. In this figure, a vector parallel or antiparallel to the $X$-axis indicates that the SV of a galaxy tends to lie in the supergalactic plane (or LSC plane). The cluster A1412, which appears elongated in the equatorial system (Fig. 1a) looks nearly face-on in the supergalactic system (Fig. 4a). This is because of the coordinate transformation.

Anisotropy can easily be seen in A1412, A2061, A2065 and $\mathrm{A} 4038$ where $\Delta_{11}>1.5 \sigma$ (Figs. $4 \mathrm{a}, \mathrm{c}, \mathrm{d}, \mathrm{g}$ and Table 3 ). The vectors tending to be parallel or antiparallel with respect to the $X$-axis $\left(B=0^{\circ}\right)$ dominate in A1412 and A4038, suggesting that the SVs of galaxies in these clusters tend to lie parallel to the LSC plane (see Figs. 4a, g). The SV orientations tend to be 

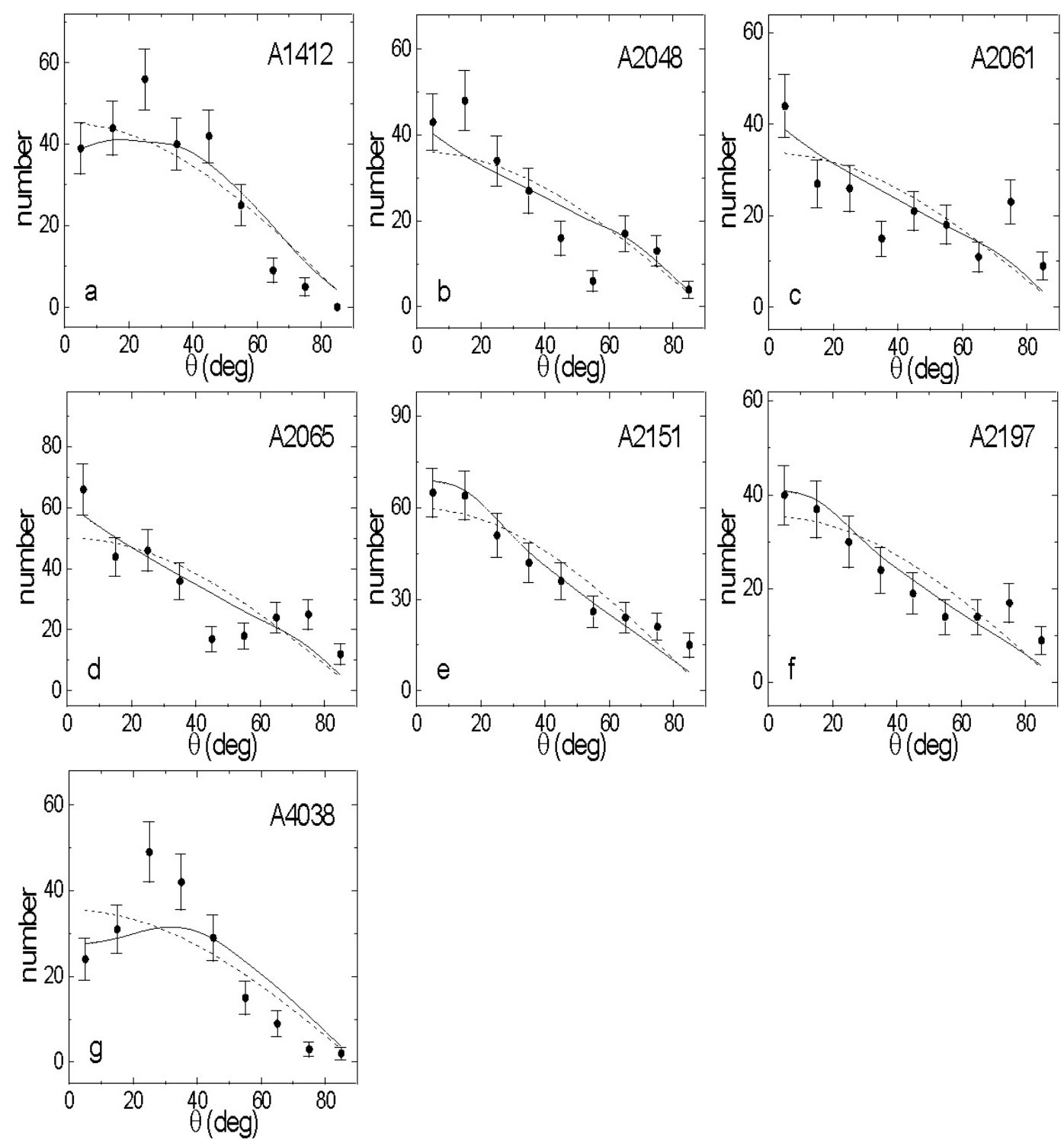

Fig. 3. The polar angle $(\theta)$ distribution of galaxies in A1412 a); A2048 b); A2061 c); A2065 d); A2151 e); A2197 f); and A4038 g). The solid line represents the expected isotropic distributions; the dashed lines give the cosine, for comparison. The observed counts with statistical $\pm \sigma$ error bars are shown. $\theta=0^{\circ}$ corresponds to the galactic SV lying in the LSC plane. Anisotropy can be seen in all figures.

oriented perpendicular to the LSC plane in A2061 and A2065 (see Figs. 4c, d).

Figure $4 \mathrm{~h}$ shows the all sky map of the clusters with their preferred alignment for $\theta$. It is found that the clusters having $B<20^{\circ}$ (A1214 and A4038) show a similar alignment despite their great separation in longitude $\left(\Delta L>180^{\circ}\right)$. In contrast to this, a similar alignment can be seen in the 4 clusters (A2061, A2065, A2151 \& A2197) having relatively small separation in both longitude $\left(\Delta L \sim 30^{\circ}\right)$ and latitude $\left(\Delta B<15^{\circ}\right)$. However, these clusters are at higher latitudes $\left(B>35^{\circ}\right)$. It is difficult to interpret this result because of the poor database. However, finding these anisotropies is a noticable result. This result may indicate that the supergalactic plane coincides with a plane of a larger concentration of galaxies at much larger scales. This should be tested in a future study.

Thus, in the polar angle distributions we found anisotropy for all 7 clusters $-\Delta_{11}$ is found to be positive in 4 cases and negative in 3 cases - which suggests that the SV orientations of galaxies in Abell clusters of BM type III tend to be oriented both parallel and perpendicular to the LSC plane.

\subsection{Anisotropy in the azimuthal angle distribution}

Figure 5 shows the distributions of the SV projections $(\phi)$ of galaxies in the 7 Abell clusters with respect to the Virgo cluster (VC) center. In the figures, $\phi=0^{\circ}$ means the direction towards the Virgo cluster center (or the LSC center).

All seven clusters of BM type III show anisotropy in the azimuthal angle $(\phi)$ distribution. The statistical results for the $\phi$-distribution of the cluster galaxies are given in Table 4 . The clusters Abell 1412, Abell 2061, Abell 2065 and Abell 4038 give 0\% chi-square and first order Fourier probablity. In these clusters the correlation coefficient $(\mathrm{C})$ is found to be $>4$. 

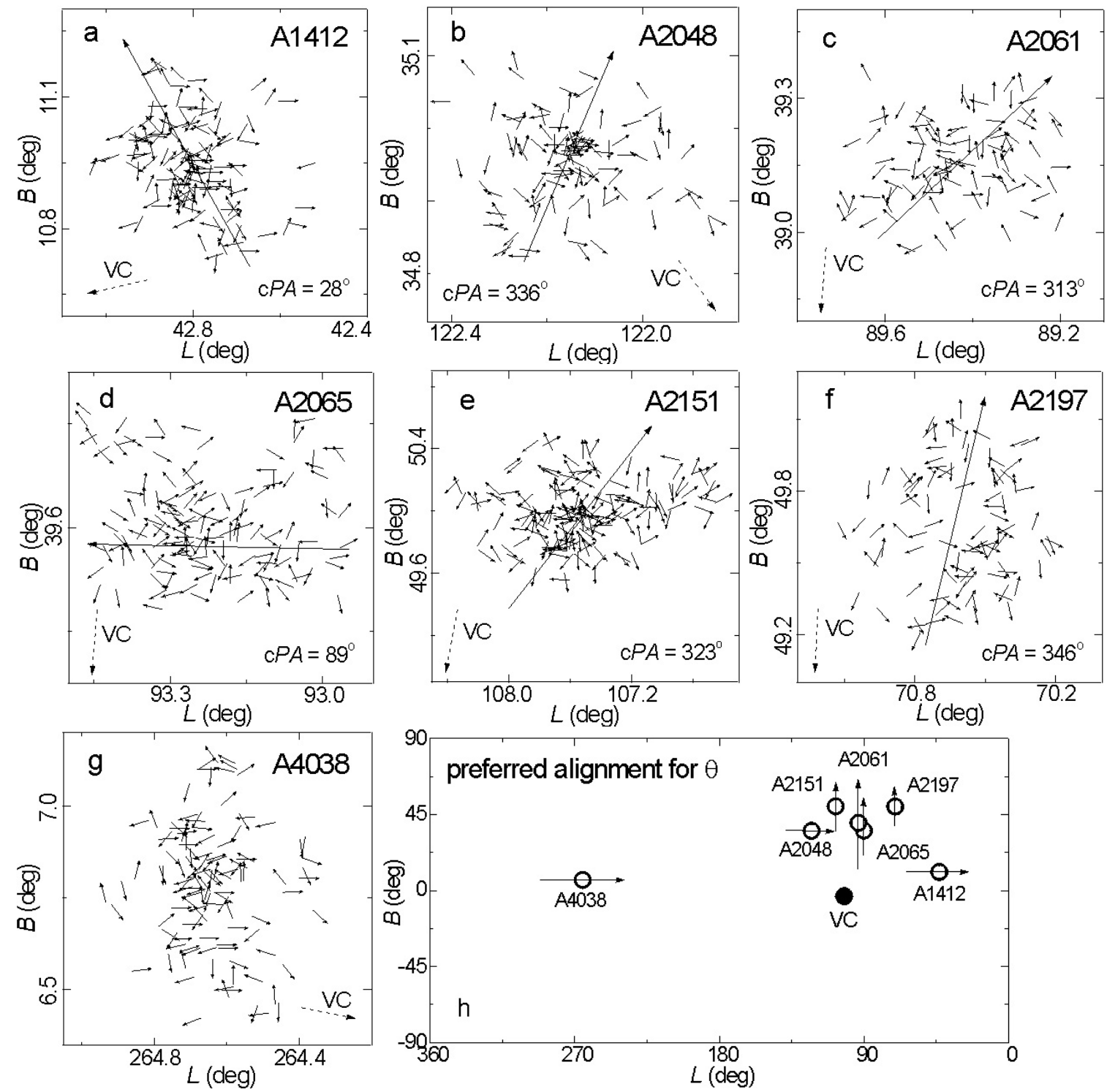

Fig. 4. The polar angle $(\theta)$ in A1412 a); A2048 b); A2061 c); A2065 d); A2151 e); A2197 f); and A4038 g). A vector represents the angle $\theta$ of a galaxy positioned at the start of an arrow. The magnitude of the vector is kept constant. The solid and dashed line with an arrow represent the supergalactic PA of the cluster ( $\mathrm{cPA}$ ) and the direction towards the Virgo cluster (VC) center $\left(L=102^{\circ} .26, B=-3^{\circ} .25\right)$. A vector perpendicular to the $X$-axis indicates that the SV of galaxy tends to be oriented perpendicular to the LSC plane. Figure 4h represents an all sky plot of preferred orientations of $\theta$. The solid line with an arrow represents the preferred alignment: parallel or perpendicular to the LSC plane. The magnitude of these lines gives the relative strength of anisotropy: the larger the stronger.

In the histogram of the $\phi$-distribution Abell 1412 and Abell 2048 show humps in the middle range (at $-35^{\circ}(1.5 \sigma)$, $-25^{\circ}(3 \sigma),-15^{\circ}(1.5 \sigma)$ for Abell 1415 and at $-5^{\circ}(1.5 \sigma)$, $-15^{\circ}(>2 \sigma)$ for Abell 2048). In Abell 2048 dips at $-85^{\circ}$ $(1.5 \sigma)$ and $-75^{\circ}(1.5 \sigma)$ also can be seen (Figs. 5a, b). Due to these humps and dips the $\Delta_{11}$ values are found to be positive at a $2.6 \sigma$ and $1.8 \sigma$ level in Abell 1412 and Abell 2048, respectively. This result suggests that the SV projections of galaxies in these clusters tend to point towards the Virgo cluster center.

The five clusters Abell 2061, Abell 2065, Abell 2151, Abell 2197 and Abell 4038 show identical orientation in the $\phi$-distribution with respect to the LSC center (or the Virgo cluster center). In these clusters the $\Delta_{11}$ value is found to be negative (Table 4 ). In the $\phi$-distribution, the $\Delta_{11}$ value would be negative for $\phi$ when humps are at $-90^{\circ}$ to $-50^{\circ}$ (first 4 bins) and at $50^{\circ}$ to $90^{\circ}$ (last 4 bins) and dips are at $-50^{\circ}$ to $+50^{\circ}$. In Figs. $5 \mathrm{c}, \mathrm{d}, \mathrm{e}, \mathrm{f}, \mathrm{g}$, dips in the middle range $\left(-15^{\circ}\right.$ to $25^{\circ}$ for Abell $2061,5^{\circ}$ to $45^{\circ}$ for Abell 2065 , at $-25^{\circ},-15^{\circ}$ and $25^{\circ}$ for Abell 2151, at $15^{\circ}, 25^{\circ}$ for Abell 2197 , and at $5^{\circ}, 15^{\circ}$ for Abell 4038) can be seen. In addition to these dips, humps at $-85^{\circ}$, $-55^{\circ}$ for Abell 2061, at $-75^{\circ}$ for Abell 2151, and at $65^{\circ}, 75^{\circ}$ for Abell 4038 can be seen. These dips and humps cause the $\Delta_{11}$ value to be negative. A negative $\Delta_{11}$ suggests that the SV projections of galaxies tend to point perpendicular to the Virgo cluster center.

Figures $6 \mathrm{a}-\mathrm{g}$ shows the plot of the SV projections of galaxies in the investigated clusters. In this figure, a vector pointing towards the VC center indicates that the SV projection of 

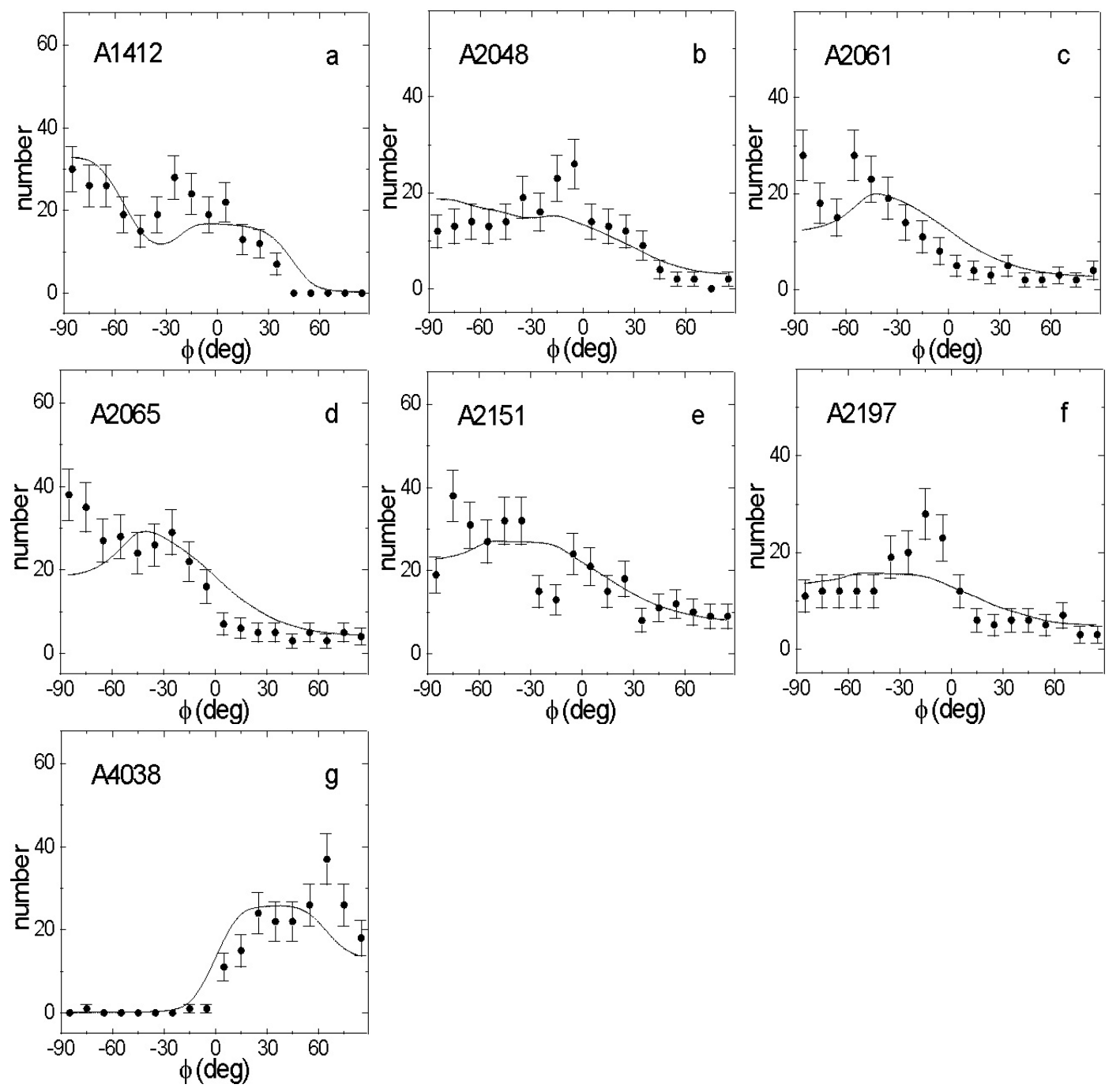

Fig. 5. The azimuthal angle ( $\phi$ ) distribution of galaxies in A1412 a); A2048 b); A2061 c); A2065 d); A2151 e); A2197 f); and A4038 g). $\phi=0^{\circ}$ means the direction to the LSC center or the Virgo cluster center. Anisotropy can be seen in all figures. Symbols as in Fig. 3.

Table 4. Statistics of the azimuthal angle distribution of galaxies in 7 Abell clusters. See Table 3 for an explanation of the columns.

\begin{tabular}{ccccc}
\hline \hline Abell & $P\left(>\chi^{2}\right)$ & $C / C(\sigma)$ & $\Delta_{11} / \sigma\left(\Delta_{11}\right)$ & $P\left(>\Delta_{1}\right)$ \\
\hline 1412 & 0.000 & +5.4 & +2.6 & 0.000 \\
2048 & 0.035 & +3.1 & +1.7 & 0.031 \\
2061 & 0.000 & +4.3 & -4.5 & 0.000 \\
2065 & 0.000 & +8.5 & -4.6 & 0.000 \\
2151 & 0.038 & +1.2 & -1.2 & 0.110 \\
2197 & 0.032 & +4.2 & -1.4 & 0.041 \\
4038 & 0.000 & +5.1 & -5.2 & 0.000 \\
\hline
\end{tabular}

that galaxy tends to point towards the VC center. Anisotropy can easily be seen in A1412, A2061, A2065 and A4038 where $\Delta_{11}>2.5 \sigma$ (Figs. 6a, c, d, g and Table 4). The vectors pointing towards the VC center dominate in A1412, suggesting that the SV projections of galaxies tend to point towards the Virgo cluster (see Fig. 6a). An opposite orientation can be seen in A2061, A2065 and A4038 (see Figs. 6c, d, g).
Figure 6h shows the all sky map of the clusters with their preferred alignment for $\phi$. It is found that the two farthermost clusters $\left(R V>25000 \mathrm{~km} \mathrm{~s}^{-1}\right) \mathrm{A} 1412$ and A2048 show a similar alignment: SV projections of galaxies in these clusters tend to point towards the Virgo cluster center. The other clusters including the nearby cluster A4038 $\left(R V<10000 \mathrm{~km} \mathrm{~s}^{-1}\right)$ in our database show an opposite alignment. The nearby cluster (A4038) shows the strongest $\left(\Delta_{11}>5 \sigma\right)$ alignment in the $\phi$-distribution. This is an important result to define a relation between the Local Supercluster and the distant cluster. This result should be verified using a richer database in the future.

Thus, in the azimuthal angle distributions all 7 clusters showed anisotropy - the $\Delta_{11}$ is found to be negative in 5 cases and positive in 2 cases - which suggests that the SV projections of galaxies in Abell clusters of BM type III tend to be oriented perpendicular to the LSC center (or the Virgo cluster center).

\subsection{Comparison with other work}

Thompson (1976) analysed the PA-distribution of 114 galaxies in Abell 2197 and found anisotropy whereas 

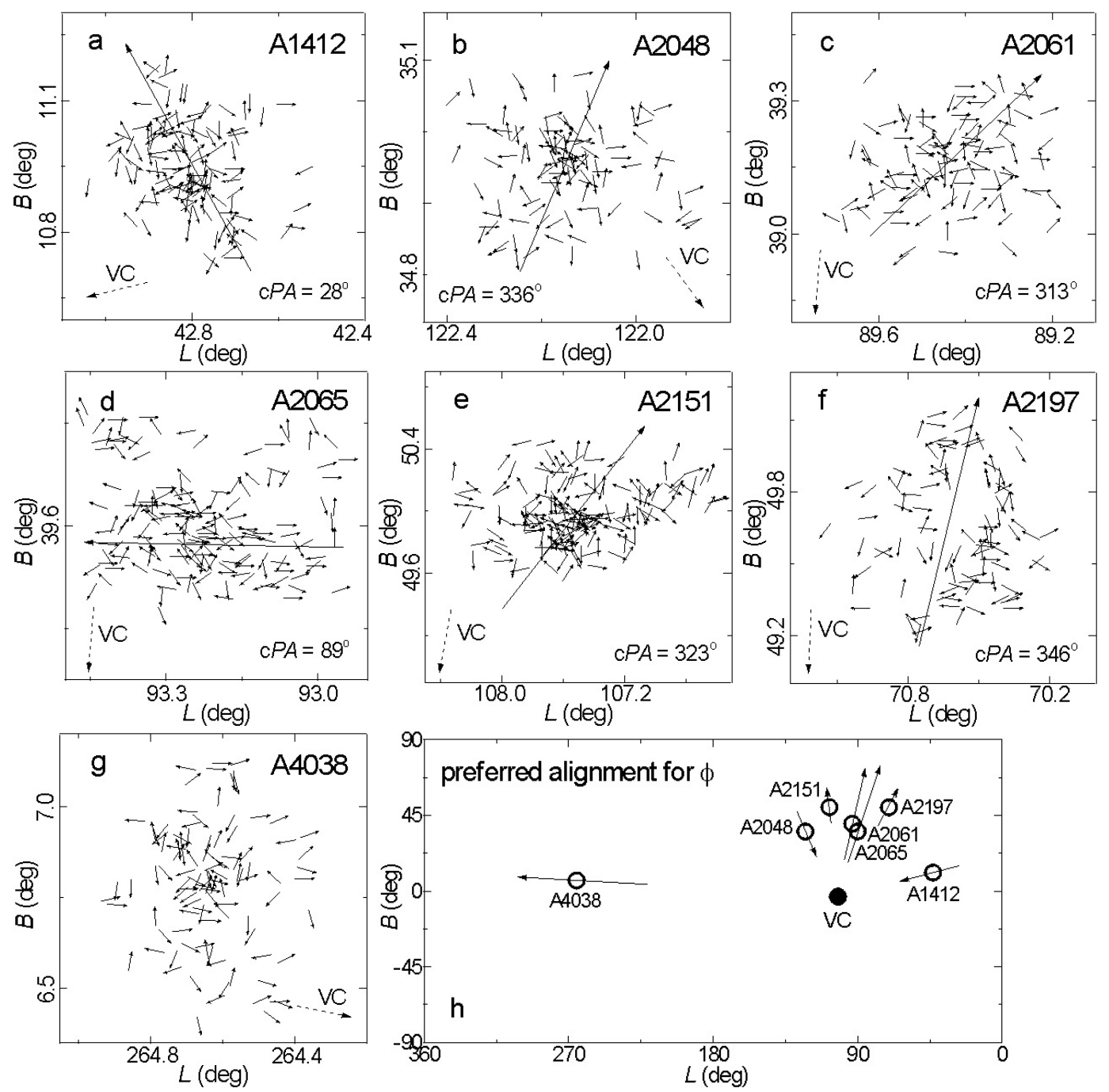

Fig. 6. The plot of the azimuthal angle ( $\phi$ ) in A1412 a); A2048 b); A2061 c); A2065 d); A2151 e); A2197 f); and A4038 g). A vector pointing towards the Virgo cluster (VC) center indicates that the SV projection of a galaxy tends to point towards the Virgo center. Figure $6 \mathrm{~h}$ represents an all sky preferred orientation plot for $\phi$. The solid line with an arrow represents the preferred alignment: pointing away or towards the Virgo cluster center. Symbols as in Fig. 4.

Adams et al. (1980) analysed 58 galaxies of the same cluster and found isotropy. We found isotropy when analysing PAdistribution of 102 galaxies in this cluster. So, our results agree with Adams et al. (1980) in the PA-distribution. However, we found anisotropy in the polar and azimuthal angle distributions of galaxies in Abell 2197. It is found that the SVs of galaxies in Abell 2197 tend to be oriented perpendicular to the LSC plane and their projections tend to point perpendicular to the Virgo cluster center.

Thompson (1976) found isotropy when analysing PA-distribution of 115 galaxies in Abell 2151. Our result does not agree with Thompson's result. We found anisotropy when analysing PA-distributions of 172 galaxies in this cluster. This disagreement might be due to the enlarged (50\% more) database. In a three-dimensional analysis, our result for this cluster is similar to Abell 2197. The SVs of galaxies in Abell 2151 tend to be oriented perpendicular to the LSC plane and their projections tend to point perpendicular to the Virgo cluster center.

\section{Conclusion}

We have analysed the spin vector orientations of 851 galaxies in 7 Abell clusters of BM type III and found anisotropy in both the polar and azimuthal angle distributions. Finding these anisotropies with respect to the Local Supercluster is a noticable result. Probably, this result could indicate that the supergalactic plane coincides with a plane of a larger concentration of galaxies at much larger scales.

It seems that the anisotropy increases systematically in galaxy alignments from early-type (BM type I, see Paper I) to late-type (BM type III) clusters. In previous work we found isotropy in 3 early-type clusters. In this work no isotropy is found.

It is found that the spin vector orientations of galaxies in the investigated Abell clusters of BM type III tend to lie both parallel and perpendicular to the LSC plane.

Our next goal is to understand the relation between the physical reference system of the investigated clusters and the 
LSC system. In addition, we intend to extend this study by analysing spin vector orientations of galaxies in the BM type intermediate (I-II or II-III) clusters in the future.

Acknowledgements. We have great pleasure to thank referee E. Battaner for his constructive criticism and useful comments. B. Aryal acknowledges the Oesterr. Akad. Austauschdienst (OeAD), Austria, for providing a scholarship and Tribhuvan University, Kathmandu, Nepal, for the study leave. We thank Y. Dixit for her help regarding the data reduction.

\section{References}

Abell, G. O., Corwin, H. G., \& Olowin, R. P. 1989, ApJS, 70, 1 (ACO) Adams, M. T., Strom, K. M., \& Strom, S. E. 1980, ApJ, 238, 445

Andernach, H., \& Andreazza, C. M. 1990, Rev. Mexicana Astron. Astrof., 21, 136

Aryal, B., \& Saurer, W. 2000, A\&A, 364, L97

Aryal, B., \& Saurer, W. 2001, in Galaxy Disk and Disk Galaxies, ed. J. S. Funes \& E. M. Corsini, ASP Conf. Ser., 230, 145

Aryal, B., \& Saurer, W. 2004, A\&A, 425, 871 (Paper I)

Aryal, B., \& Saurer, W. 2005, A\&A, 432, 431

Baier, F. W., Godlowski, W., \& MacGillivray, H. T. 2003, Ap\&SS, 403, 847

Bautz, L. P., \& Morgan, W. W. 1970, ApJ, 162, L149

de Vaucouleurs, G., de Vaucouleurs, A., Corwin, H. G., et al. 1991, Third Reference Catalogue of Bright Galaxies (New York: Springer-Verlag)
Doroshkevich, A. G. 1973, ApJ, 14, L11

Flin, P., \& Godlowski W. 1986, MNRAS, 222, 525

Fouque, P., \& Paturel, G. 1985, A\&A, 150, 192

Godlowski, W. 1993 MNRAS, 265, 874

Godlowski, W. 1994 MNRAS, 271, 19

Hu, F. X., Wu, G. X., Su, H. J., \& Liu, Y. Z. 1995, A\&A, 302, 45

Kashikawa, N., \& Okamura, S. 1992, PASJ, 44, 493

Lauberts, A. 1982, ESO/Uppsala Survey of the ESO B Atlas. ESO, Garching bei Muenchen

Nilson, P. 1973 Uppsala General Catalogue of Galaxies, Nova Acta Uppsala University, Ser. V.A., Vol. 1

Ozernoy, L. M. 1978, in The Large Scale Structure of the Universe, ed. M. S. Longair, \& J. Einasto (Dordrecht: Reidel), Proc. IAU Symp., 79, 427

Peebles, P. J. E. 1969, ApJ, 155, 393

Rhee, G. F. R. N., \& Ross, N. 1989, Ap\&SS, 157, 201

Rhee, G. F. R. N., van Haarlem, M. P., \& Katgert, P. 1991, A\&AS, 91, 513

Tammann, G. A., \& Sandage, A. 1976, ApJ, 207, L1

Thompson, L. A. 1976, ApJ, 209, 22

van den Bergh, S. 1978, Ap\&SS, 53, 415

van Kampen, E., \& Rhee, G. F. R. N 1990, A\&A, 237, 283

Vorontsov-Vel, Y. B. A., Krasnogorskaya, A. A., \& Arkhipova, V. P. 1962-74, in Morphological Catalogue of Galaxies (Part I-V), Trudy Gosud. Astron. Inst. Shternberga 